\title{
Field-Induced Magnetic Monopole Plasma in Artificial Spin Ice
}

\author{
M. Goryca, ${ }^{1}$ X. Zhang, ${ }^{2}$ J. Li®${ }^{1}{ }^{1}$ A. L. Balk, ${ }^{1}$ J. D. Watts, ${ }^{3,4}$ C. Leighton, ${ }^{3}$ \\ C. Nisoliø ${ }^{5}$ P. Schiffer $\oplus^{2,6}$ and S. A. Crooker ${ }^{1}$ \\ ${ }^{1}$ National High Magnetic Field Lab, Los Alamos National Laboratory, \\ Los Alamos, New Mexico 87545, USA \\ ${ }^{2}$ Department of Applied Physics, Yale University, New Haven, Connecticut 06520, USA \\ ${ }^{3}$ Department of Chemical Engineering and Materials Science, University of Minnesota, \\ Minneapolis, Minnesota 55455, USA \\ ${ }^{4}$ School of Physics and Astronomy, University of Minnesota, Minneapolis, Minnesota 55455, USA \\ ${ }^{5}$ Theoretical Division, Los Alamos National Laboratory, Los Alamos, New Mexico 87545, USA \\ ${ }^{6}$ Department of Physics, Yale University, New Haven, Connecticut 06520, USA
}

(Received 10 August 2020; revised 15 December 2020; accepted 6 January 2021; published 2 March 2021)

Artificial spin ices (ASIs) are interacting arrays of lithographically defined nanomagnets in which novel, frustrated magnetic phases can be intentionally designed. A key emergent description of fundamental excitations in ASIs is that of magnetic monopoles-mobile quasiparticles that carry an effective magnetic charge. Here, we demonstrate that the archetypal square ASI lattice can host, in specific regions of its magnetic phase diagram, plasmalike regimes containing a high density of mobile magnetic monopoles. These regimes result from the magnetic field-tunable tension on the Dirac strings connecting mobile monopoles. By passively "listening" to spontaneous monopole noise under conditions of strict thermal equilibrium, we reveal their intrinsic dynamics and show that monopole kinetics are most diffusive (that is, minimally correlated) in the plasma regime. These results open the door to on-demand monopole regimes having continuously field-tunable densities and dynamic properties, thereby providing a new paradigm for probing the physics of effective magnetic charges in synthetic matter.

DOI: 10.1103/PhysRevX.11.011042

Owing to their user-defined geometries of interacting magnetic elements, artificial spin ices (ASIs) provide a highly flexible and powerful platform with which to investigate the rich physics of frustrated spin systems [1-3]. Initially conceived [4] as two-dimensional analogs of "natural" pyrochlore spin ices $[5,6]$ such as $\mathrm{Ho}_{2} \mathrm{Ti}_{2} \mathrm{O}_{7}$, investigations of ASIs now extend well beyond these original goals and enable detailed studies of a vast selection of possible interacting lattice arrangements, including exotic magnetic topologies not found in nature [7-10]. Together with natural spin ice materials, one of their most exciting properties is that the fundamental excitations in many ASIs have a natural emergent description in terms of "effective magnetic monopoles" [11,12]—that is, mobile quasiparticles that possess the equivalent of a net magnetic charge. These charge excitations can interact with each other and with applied magnetic fields via the magnetic

Published by the American Physical Society under the terms of the Creative Commons Attribution 4.0 International license. Further distribution of this work must maintain attribution to the author(s) and the published article's title, journal citation, and DOI.
Subject Areas: Condensed Matter Physics, Magnetism, Nanophysics analog of the ubiquitous electronic Coulomb interaction, representing the emergence of a range of novel phenomena [1,2], including the possibility of "magnetricity" [12].

While the presence of monopoles in ASI [13] has been observed in pioneering imaging measurements [14-16], dynamical studies of monopole kinetics [17-19], and (especially) the ability to tune continuously through monopole-rich regimes in thermal equilibrium, remain at an early stage. Because their underlying magnetic interactions can be engineered to manifest near room temperature, ASIs are particularly well suited to studies of monopole dynamics and other collective modes. In this work, we use a high-bandwidth magneto-optical noise spectrometer to passively detect spontaneous magnetization fluctuations in thermally active square ASI. Because fluctuations of the constituent magnetic elements in ASIs are inextricably linked to the kinetics of monopoles, the system's broadband magnetization noise spectrum-in thermal equilibrium - naturally encodes the intrinsic timescales and dynamic correlations of the underlying monopole excitations. The noise reveals specific regions in the field-dependent phase diagram where the density of mobile monopoles increases well over an order of magnitude compared with neighboring regimes, a consequence of 
the field-tunable tension on the Dirac strings connecting mobile monopoles. Moreover, detailed noise spectra demonstrate that monopole kinetics are minimally correlated (i.e., most diffusive) in this plasmalike regime. Discovery of on-demand monopole regimes with tunable kinetic properties opens the door to new probes of magnetic charge dynamics and provides a new paradigm for studies of magnetricity in artificial magnetic materials.

We consider the prototypical square ASI lattice [4], shown in Fig. 1(a). Each ferromagnetic nanoisland behaves as a single Ising-like macrospin with net magnetization parallel or antiparallel to its long axis due to shape anisotropy. Crucially, the islands are made sufficiently thin so that they are superparamagnetic and thermally active at room temperature [17,20-23]; i.e., in the absence of strong biasing magnetic fields, each island's magnetization can fluctuate spontaneously. These fluctuations ensure the lattice can efficiently sample the vast manifold of possible moment configurations and remain near its magnetic ground state in thermal equilibrium.

Our study focuses on a previously unexplored characteristic of thermal square ASI: namely, that its fielddependent magnetic phase diagram and ground-state moment configuration must include regions where monopole excitations play a dominant and active role, even under conditions of strict thermal equilibrium. This requirement can be understood by considering the relative energies of the four possible vertex types (I-IV), shown and described in Fig. 1(b) in order of increasing energy at zero applied magnetic field. At zero field, the ground state of square ASI is an ordered antiferromagnetic tiling of type-I vertices $[20,21,24]$, which obey the "2-in-2-out" ice rule and do not possess any net polarization. However, for sufficiently large in-plane magnetic fields applied at angles near a lattice diagonal $\left( \pm 45^{\circ}\right)$, one of the four possible type-II vertices must be energetically favored. Type-II vertices also obey ice rules but possess a net polarization. The field-dependent phase diagram of thermal square ASI should then qualitatively resemble the schematic drawn in Fig. 1(c).

The crossover between type-I and type-II magnetic order (or between type-II orderings with different polarization) obviously requires the reversal of individual islands. Crucially, as depicted in Fig. 1(d), flipping any island within either a type-I or type-II ordered lattice unavoidably creates a pair of higher-energy type-III vertices, which have 3 -in-1-out or 3-out-1-in moment configurations and therefore possess an effective magnetic "charge" that can be regarded as a magnetic monopole quasiparticle excitation [1-3]. Subsequent flips of other islands can create additional monopole pairs, annihilate adjacent monopole pairs, or cause an existing monopole to move through the ASI

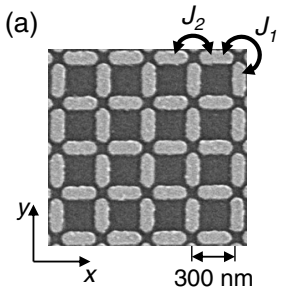

(d)

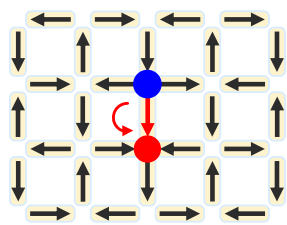

$I+I \rightarrow I I I+I I$

(Monopole creation)
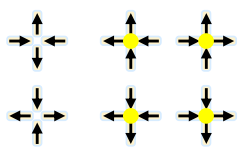

Type-I Type-II

(e)

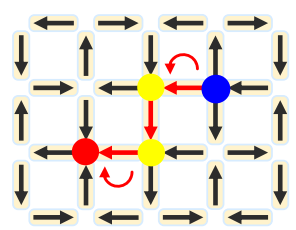

$\mathrm{I}+\mathrm{III} \rightarrow \mathrm{III}+\mathrm{II}$

(Monopole motion) (c)
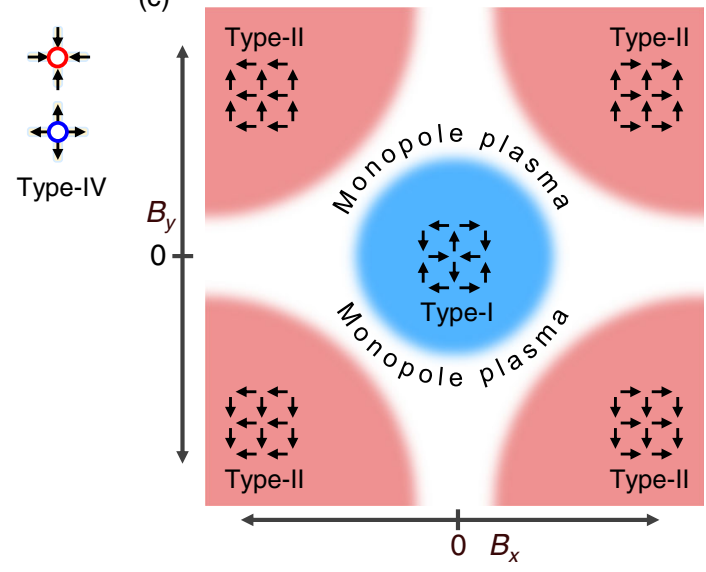

FIG. 1. Field-dependent phase diagram of thermally active square ASI. (a) Scanning electron microscopy image of the sample. Each $\mathrm{Ni}_{0.8} \mathrm{Fe}_{0.2}$ island has lateral dimensions of $220 \mathrm{~nm} \times 80 \mathrm{~nm}$ and thicknesses of $3.5 \mathrm{~nm}$, and behaves as a single superparamagnetic Ising moment that, in the absence of any biasing field, exhibits rapid thermodynamic fluctuations near room temperature. (b) Four vertex types in archetypal square ASI, in order of increasing energy at zero applied magnetic field. Type-I vertices have the lowest energy because the nearest-neighbor dipolar coupling $J_{1}$ exceeds the next-nearest-neighbor coupling $J_{2}$. Types-I and -II vertices have 2-in-2-out configurations and therefore obey ice rules (but only type-II vertices have a net polarization), while type-III vertices have 3-in-1-out or 3out-1-in configurations and therefore have a monopole effective magnetic "charge." (Type-IV vertices also have magnetic charge but are energetically very unfavorable and only rarely occur.) (c) Notional schematic of the anticipated field-dependent phase diagram of square ASI, showing the two well-defined ground states of the system: full tiling with type-I vertices at small applied fields $B_{x, y} \approx 0$, and polarized type-II vertex tiling when $\left|B_{x}\right|$ and $\left|B_{y}\right|$ are both large. Near the boundaries, the equilibrium dynamics are determined by the thermal creation, annihilation, and motion of type-III monopole vertices, which generate magnetization noise. (d) Schematic showing how a thermal fluctuation in the type-I phase creates a pair of type-III monopole vertices. (e) Subsequent fluctuations can cause the monopoles to diffuse, leaving behind a Dirac string of type-II (yellow) vertices. Red arrows show islands that have flipped; blue and red dots indicate the mobile monopole vertices. 
lattice [Fig. 1(e)]. Within a type-I (type-II) ordered region, the creation and subsequent separation of a monopole pair along a staggered diagonal direction creates a string of type-II (type-I) vertices [17]. As discussed in detail below, for applied magnetic fields $B_{x}$ and $B_{y}$ that place the system near the boundaries between type-I and type-II ordering, these monopole excitations, once thermally created, can be expected to diffuse freely along specific diagonal directions determined by the applied field, with no cost in energy [25], because the type-I and type-II vertex energies are degenerate along the boundary. Therefore, we may expect thermal square ASI to host field-tunable regimes of mobile magnetic monopoles [see Fig. 1(e)].

To search for dynamic monopole regimes in square ASI and to quantify their timescales and correlations-all under conditions of strict thermal equilibrium - we developed a broadband magnetization noise spectrometer to measure

(a)

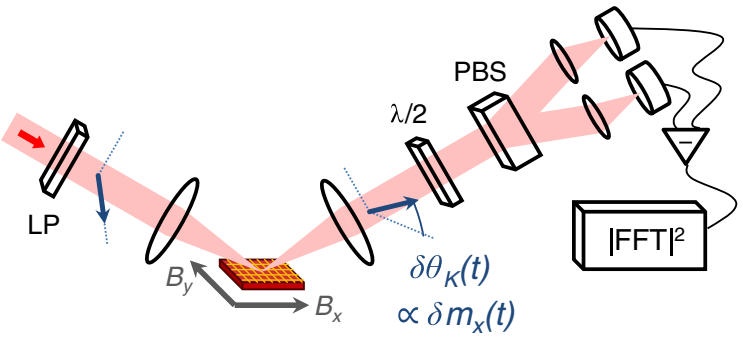

(b)

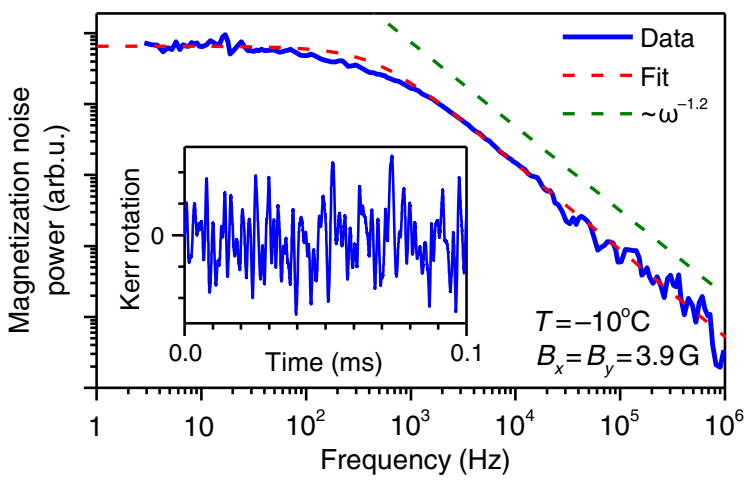

FIG. 2. Optical detection of broadband magnetization noise in ASI. (a) Experimental schematic: A weak probe laser is linearly polarized (LP) and focused on the ASI. Magnetization fluctuations of the horizontal islands, $\delta m_{x}(t)$, impart Kerr rotation fluctuations $\delta \theta_{K}(t)$ on the reflected laser, which are detected with a polarization beamsplitter (PBS) and balanced photodiodes. The frequency spectrum of the magnetization noise power, $P(\omega)$ equivalent to the Fourier transform of the temporal magnetization correlation function $\left\langle\delta m_{x}(0) \delta m_{x}(t)\right\rangle$-is computed and averaged in real time. (b) Example of characteristic magnetization noise from square thermal ASI in the time domain (inset), and in the corresponding frequency domain spanning nearly 6 orders of magnitude from $1 \mathrm{~Hz}$ to $1 \mathrm{MHz}$. The dashed red line shows a fit to the model described in the main text, and the dashed green line shows that the noise decays as a power law at high frequencies, in this case as $P \sim \omega^{-1.2}$, which indicates correlated and subdiffusive kinetics. the frequency spectrum of the system's intrinsic magnetization fluctuations [see Fig. 2(a) and the Appendix]. Samples were mounted in the $x-y$ plane, with horizontal and vertical islands oriented along $\hat{x}$ and $\hat{y}$. A weak probe laser was linearly polarized and focused on the ASI. Because of the longitudinal magneto-optical Kerr effect (MOKE), magnetization fluctuations in the $\hat{x}$ direction, $\delta m_{x}(t)$, imparted Kerr rotation fluctuations $\delta \theta_{K}(t)$ on the polarization of the reflected laser, which were detected with balanced photodiodes. This "magnetization noise" was digitized, and its frequency-dependent power spectrum $P(\omega)$ was computed and signal-averaged in real time. Figure 2(b) shows a characteristic spectrum measured out to $1 \mathrm{MHz}$, spanning over 5 orders of magnitude in both frequency and detected noise.

We first analyze the noise from a control lattice that contains only horizontal islands oriented along $\hat{x}$. Figure 3(a) shows a map of the measured total (frequency-integrated) noise power versus applied in-plane magnetic fields $B_{x}$ and $B_{y}$. As expected, significant noise was only observed when $B_{x} \approx 0$, where the thermally active islands were unbiased and fluctuated freely. For larger $\left|B_{x}\right|$, the islands were polarized and effectively frozen, suppressing fluctuations. Note that $B_{y}$ has no effect because this control sample lacks vertical islands and because the Ising-like magnetization of the horizontal islands is not influenced by $B_{y}$ in this small range $( \pm 7 \mathrm{G})$.

In marked contrast, Fig. 3(b) shows the noise map from square ASI, where strong dipolar interactions between nearest-neighbor (adjacent vertical and horizontal) islands lead to the stable type-I magnetic ordering sketched in the phase diagram of Fig. 1(c). Indeed, the noise map exhibits a large, dark, central region when $B_{x, y} \approx 0$, indicating suppressed fluctuations, as expected for stable type-I order. Towards the four corners of the map, where both $\left|B_{x}\right|$ and $\left|B_{y}\right|$ are large, the magnetization noise again vanishes, indicating field-stabilized type-II tiling. Strikingly, however, there is a bright diamond-shaped boundary region, indicating a high level of spontaneous noise between the type-I and type-II magnetic orderings.

The boundary region occurs when $\left|B_{x}\right|+\left|B_{y}\right|$ equals the crossover field $B_{c}$, where type-I vertices and type-II vertices with net moment along the field direction become exactly degenerate in energy (see Supplemental Material Fig. S1 for additional details and a plot of all vertex energies versus $B$ [26]). As discussed above, spontaneous reversal of an island within a type-I or type-II ordered region creates a pair of type-III monopole vertices [Fig. 1(d)]. The key point is that at $B_{c}$, these thermally generated monopoles can then separate and are expected to diffuse freely along the staggered diagonal path that is most closely aligned with the applied field, as illustrated in Fig. 1(e). Within this simple vertex model, this motion, denoted by the process III + I $\leftrightarrow$ II + III, has no collective energy cost along the boundary where type-I and -II vertex 


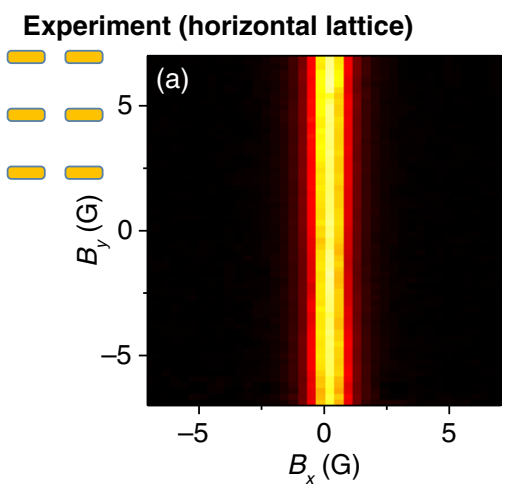

Simulation (monopole density)

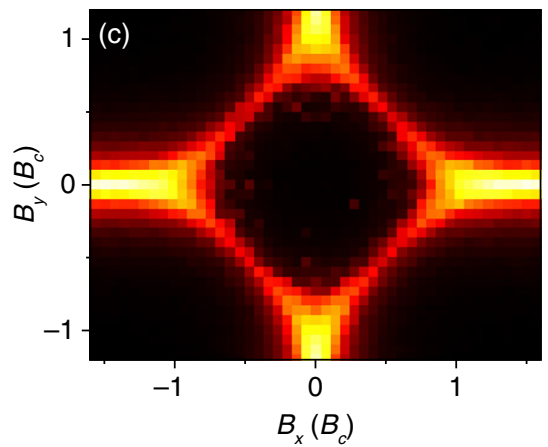

Experiment (square lattice)

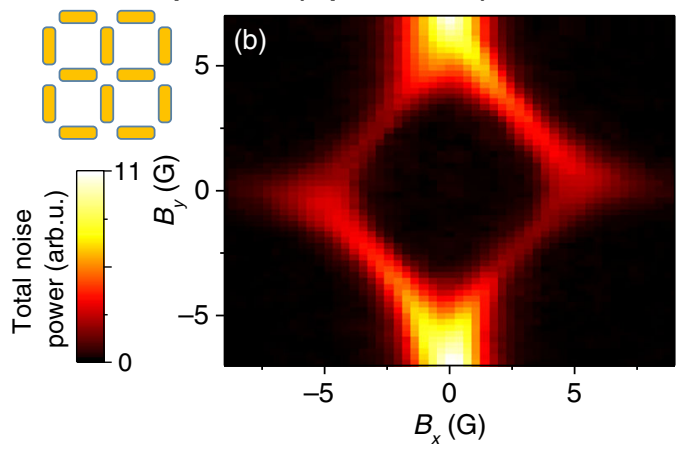

Simulation (horizontal flip rate)

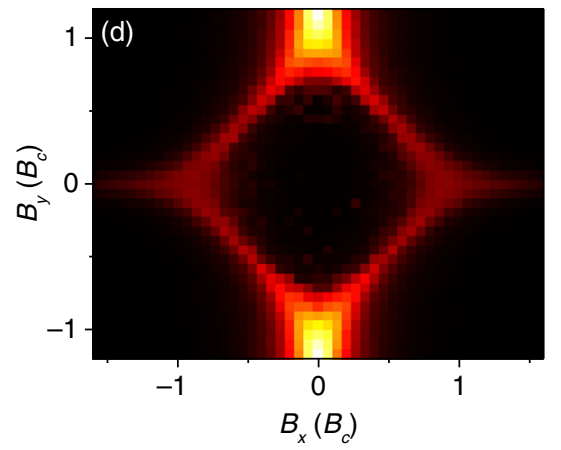

FIG. 3. Noise maps reveal the magnetic phase diagram of thermally active square ASI. (a) Map of the total magnetization noise power [ $\int P(\omega) d \omega$, integrated from $250 \mathrm{~Hz}$ to $\left.250 \mathrm{kHz}\right]$ from a control sample that contains only horizontal islands, versus applied in-plane magnetic fields $B_{x}$ and $B_{y}$. Here, $T=-10.0^{\circ} \mathrm{C}$. For $B_{x} \approx 0$, the islands fluctuate spontaneously, but fluctuations are suppressed at larger $\pm B_{x}$ where all the islands are polarized by the field. (b) Corresponding noise map from square ASI. The diamond-shaped boundary, where type-I and type-II vertices are degenerate in energy, is revealed by thermodynamic noise arising from the dynamics of type-III (monopole) vertices. The map lacks the fourfold symmetry of the square lattice because it measures only fluctuations along $\hat{x}$ but not $\hat{y}$. The very slight skewing of the diamond is likely due to imperfections in the lithography. Supplemental Material Fig. S2 shows raw timedomain noise signals at various applied fields [26]. (c) Corresponding map of the calculated density of monopole vertices from Monte Carlo spin dynamics simulations. Along the diamond-shaped boundary where the density is large, the majority of the monopoles are freely diffusing (see Fig. 4). (d) Map of the calculated rate of horizontal magnetization flips, which is directly related to the measured total noise. Measured and simulated noise maps at $T=6{ }^{\circ} \mathrm{C}$ and $23^{\circ} \mathrm{C}$, and measured noise maps for square ASI with a different lattice constant are shown in Supplemental Material Figs. S3 and S4 [26].

energies are degenerate. No net energy is required to lengthen or shorten the Dirac string of flipped vertices connecting the two monopoles-i.e., the string tension vanishes at $B_{c}[17,25]$. Most importantly, the freedom to separate leads to a substantial increase in the amount of time a monopole remains in the lattice, which therefore markedly increases the equilibrium density of mobile monopole vertices. Being topological quasiparticles, the monopole density is limited only by the rate at which they annihilate by diffusing to an edge of the lattice or by encountering monopoles of opposite charge. This special regime stands in marked contrast to the case when $\left|B_{x}\right|+\left|B_{y}\right|<B_{c}$ (or $>B_{c}$ ), where the ground-state configuration is type-I (or type-II) and where monopole-pair separation that creates additional type-II (or type-I) vertices is energetically unfavored and therefore suppressed. In both of these cases, the Dirac string has a nonzero tension that prevents monopole pairs from separating freely and favors their recombination shortly following their creation. As demonstrated below, this is accompanied by increasingly anomalous monopole diffusion and correlated dynamics.

Because the stochastic creation, annihilation, and motion of monopoles are intimately linked to the reversal of individual islands, the boundary region is clearly revealed by magnetization noise. Equivalently, all noise in square ASI is necessarily due to monopole kinetics. The diamondshaped boundary therefore signals a field-tunable regime of dynamic magnetic monopoles. We note that the noise map also shows bright vertical stripes when $B_{x} \approx 0$ and $\left|B_{y}\right|$ is large, indicating strong fluctuations at the boundary between different type-II orientations. These fluctuations arise from an effective dimensional reduction, where all the vertical islands are polarized by $B_{y}$, and thereforeconsidering nearest-neighbor coupling $J_{1}$ only-there is no energy difference if a horizontal island is magnetized along $\pm \hat{x}$. Thermal fluctuations $\delta m_{x}(t)$ therefore occur, similar to the case of the control lattice at $B_{x}=0$. 
(Weaker next-nearest-neighbor coupling $J_{2}$ is insufficient, compared to $k T$, to stabilize any order.)

To better understand the noise data and provide additional insight into the underlying mechanisms involved, we perform Monte Carlo simulations of square ASI (see Appendix). Figure 3(c) shows the calculated density of type-III monopole vertices in the ASI lattice. Figure 3(d) shows the computed total rate of horizontal spin flips, which is directly linked to the measured total noise power. Regions of high monopole density correspond to regions of maximum flip rate, which in turn corresponds very well with the measured noise map, thereby validating the connection between the measured noise power and the density of mobile monopoles.

Returning to the phase diagram sketched in Fig. 1(c), both experiments and simulations confirm that the type-I and type-II ordered regions in thermal square ASI are separated by a plasmalike regime containing a high density of mobile monopoles. Simulations indicate not only that the monopole density increases by over 2 orders of magnitude at these boundaries [Fig. 3(c)] but also that the majority of these monopoles are free (Fig. 4). Crucially, we note that both the monopole density and (as shown below) their dynamic correlations in this regime are continuously tunable by applied magnetic fields above and below $B_{c}$, which is distinct from the monopole regime recently achieved in degenerate square ASI, which uses height-offset vertical and horizontal islands [27,28] to achieve degeneracy between type-I and all four possible type-II vertices at zero field. In other words, by tuning to $B_{c}$ in conventional square ASI, we realize a regime where correlations associated with the energy costs of changing type-I and type-II vertex populations are minimized. Of course, tuning $|B|$ away from $B_{c}$ lifts the energy degeneracy of type-I and specific type-II vertices, whereupon the Dirac strings acquire tension and the monopoles can no longer diffuse freely along a lattice diagonal. In this case, the monopoles will be affected by the magnetostatic potential associated with $B$ and can be expected to exhibit different kinetics.

Noise measurements provide an effective tool to directly probe kinetic correlations of monopoles, via the detailed shape of the noise spectrum over a very broad frequency range (typically from $1 \mathrm{~Hz}$ to $1 \mathrm{MHz}$ ). This range directly accesses the relevant intrinsic timescales of fluctuations in our thermally active ASIs and naturally complements powerful imaging techniques, such as PEEM (photoemission electron microscopy) and MFM (magnetic force microscopy), that provide excellent spatial information but are typically limited to much slower (about $0.1-0.001 \mathrm{~Hz}$ ) timescales and are less amenable to measurements in applied magnetic fields. We note that an analogous approach based on SQUID magnetometry was recently applied to $\mathrm{Dy}_{2} \mathrm{Ti}_{2} \mathrm{O}_{7}$ crystals by Dusad et al. [29], yielding important insights into monopoles in natural pyrochlore spin ices at cryogenic temperatures.

A typical noise spectrum $P(\omega)$ from square ASI is shown in Fig. 2(b). Here, $P(\omega)=\left\langle a(\omega) a^{*}(\omega)\right\rangle$, where

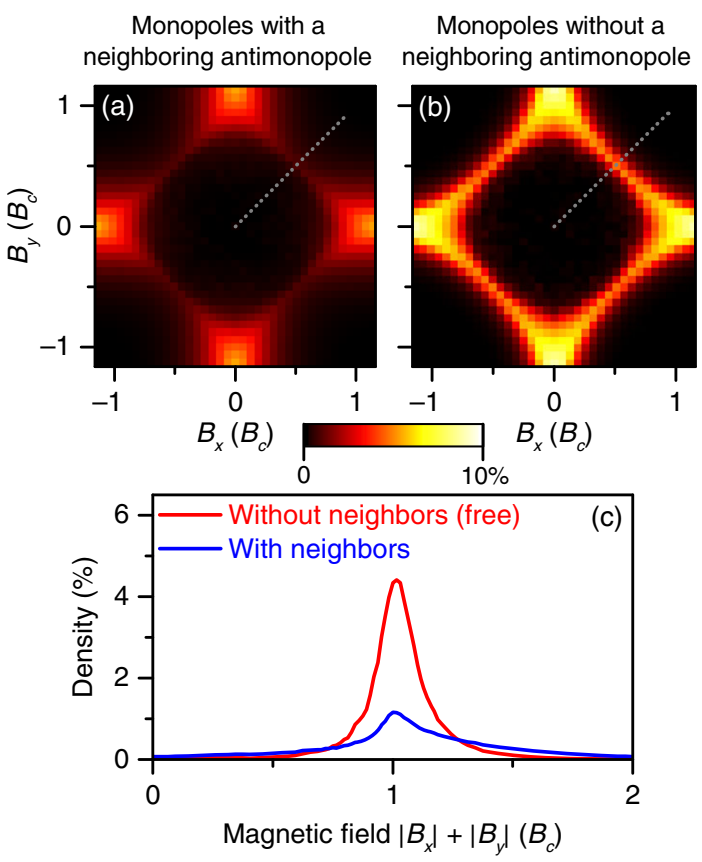

FIG. 4. Calculated density maps of monopoles both with and without an oppositely charged monopole on a neighboring vertex, vs in-plane magnetic field, from Monte Carlo simulations. (a) Map of the density of type-III monopole vertices that have a neighboring type-III vertex of opposite magnetic charge vs magnetic field, simulated at $T=1.2 J_{2} / k$. (b) Corresponding map of monopoles that do not have a neighboring monopole of opposite charge. These monopoles are more common when $B \approx$ $B_{c}$ and the Dirac string tension vanishes because thermally generated monopoles can separate and diffuse freely. (c) Densities of these two classes of monopoles vs magnetic field applied along a lattice diagonal direction [i.e., along the dotted lines in panels (a) and (b)]. In the monopole plasma regime (i.e., near the crossover field $B_{c}$ where type-I and type-II vertices are energetically degenerate), the total monopole density increases mainly due to the huge increase in the number of free monopoles, which in turn is due to the absence of Dirac string tension at $B_{c}$. Note that away from $B_{c}$, the nonzero Dirac string tension favors keeping thermally generated monopole pairs close together, and therefore, there is a higher likelihood that monopoles will be found in close proximity to one another (compare blue and red curves). By comparing simulations and data, we estimate that a monopole density of approximately $10 \%$ is realized in our experiments.

$a(\omega)$ is the Fourier transform of the noise signal $\delta m(t)$ and the brackets indicate an average over repeated measurements. Equivalently, $P(\omega)$ is the Fourier transform of the system's temporal correlation function $\langle\delta m(0) \delta m(t)\rangle$. Empirically, we find that all the measured noise spectra can be fit reasonably well by the functional form

$$
P(\omega) \propto \frac{1}{\left(\omega^{2}+\omega_{0}^{2}\right)^{\beta / 2}},
$$

where $\omega_{0}$ is a characteristic relaxation rate and $\beta$ is a powerlaw decay exponent where $P(\omega) \propto \omega^{-\beta}$ at high frequencies. 
Importantly, $\beta$ defines the "color" of the noise and is an indicator of correlated dynamics. In general, if processes responsible for magnetization dynamics are uncorrelated in time, then $\langle\delta m(0) \delta m(t)\rangle$ decays exponentially $\left(\propto e^{-t / \tau_{0}}\right)$ with a characteristic relaxation time $\tau_{0}=1 / \omega_{0}$. Per the fluctuation-dissipation theorem, the corresponding noise spectrum then exhibits a Lorentzian line shape $(\beta=2)$ and is said to be Brownian. Noise exhibiting $\beta=2$ therefore implies simple diffusive Brownian kinetics, such as from a trivial random walk with independent increments, or from monopole creation and annihilation models described, e.g., by Ryzhkin [30] and Klyuev [31]. In contrast, noise exhibiting $\beta<2$ indicates kinetics that cannot be described by a single exponential time scale [32]. Although it is always possible to simulate any $\beta$ by summing a suitable distribution of Lorentzians, for the case of ASI, the observation of $\beta<2$ can be considered in terms of anomalous monopole diffusion arising from correlated kinetics $[33,34]$. In particular, when temporal fluctuations are no longer independent but instead exhibit negative correlations (e.g., if prior fluctuations increased the magnetization, then the next fluctuation is more likely to decrease it), then monopoles will exhibit subdiffusive behavior - a particular type of anomalous diffusion where the mean-square displacement grows more slowly than linearly in time.

Such anomalous diffusion processes are related to "fractional" Brownian motion [35] and are said to retain a memory because successive increments are no longer uncorrelated but instead depend on previous increments (whereas uncorrelated increments are the hallmark of purely random, diffusive motion). More generally, a confluence of developments in recent years $[36,37]$ have tied colored noise to memory kernels [33,34] and to incremental correlations or anticorrelations that lead to anomalous diffusion. Such phenomena pertain broadly to random walks on disordered substrates, percolation clusters, and fractals [38-41], as well as to the theory of fractional random walks [35,42]. Noise following the functional form of Eq. (1) can derive from kinetics with algebraically decaying correlation functions of the form $\langle\delta m(0) \delta m(t)\rangle \propto$ $t^{-\alpha} e^{-t / \tau_{0}}$, where $\alpha=1-(\beta / 2)$. Thus, broadband noise spectroscopy can reveal both the kinetic correlations and the characteristic relaxation times as square ASI is tuned through the monopole plasma regime.

Figure 5(a) shows the dramatic evolution of $P(\omega)$ as square ASI is tuned across the boundary between type-I and type-II order, keeping $\left|B_{x}\right|=\left|B_{y}\right|$. Figure 5(b) shows the measured values of $\omega_{0}, \beta$, and the total noise power. Interestingly, although $\beta<2$ everywhere (indicating some degree of correlated dynamics, even at $B_{c}$ ), $\beta$ exhibits a clear maximum and is closest to 2 when $B=B_{c}$, where the total noise power and the monopole density are largest. This behavior indicates that memory effects are weakest in the plasmalike regime, and monopole motion along the lattice diagonal $[\mathrm{III}+\mathrm{I} \leftrightarrow \mathrm{II}+\mathrm{III}$; see Fig. 1(e)] most closely approximates ordinary Brownian diffusion. However, $\beta$ decreases when $|B| \neq B_{c}$, indicating that dynamics in square ASI become increasingly subdiffusive away from the monopole plasma regime. Given that noise in pyrochlore $\mathrm{Dy}_{2} \mathrm{Ti}_{2} \mathrm{O}_{7}$ also evinces similarly subdiffusive kinetics [29], correlated monopole dynamics in thermal equilibrium may therefore be a universal feature shared by both natural (3D) and artificial (2D) spin ices and may arise from long-range interactions or micromagnetics that are not included in our simple vertex model.

Standard Monte Carlo simulations capture very well the overall shape of the noise spectra and most of the observed trends [Figs. 5(c) and 5(d)], showing that the total noise is maximized at the monopole plasma regime at $B_{c}$. The simulated noise also exhibits a nontrivial power-law decay exponent $\beta$ in the type-I phase, which grows steeper (see dashed lines) as $B$ increases toward $B_{c}$, in agreement with the data. The simulations do not permit accurate extraction of $\beta$ at larger $B$ (i.e., into the type-II phase) because of the proximity of $\omega_{0}$ to the highest (Nyquist) frequency of the simulation, where aliasing artifacts and trivial decays due to numerics are also present.

The measured spectra [Figs. 5(a) and 5(b)] also show that the relaxation rate $\omega_{0}$ falls by orders of magnitude when tuning from type-II to the type-I phase by decreasing $|B|$. Such behavior is only partially expected: While a slowing-down of kinetics upon approaching the phase transition to long-range type-I antiferromagnetic order is expected from theory [43,44], relaxation rates are expected to increase again after entering the antiferromagnetically ordered phase - as captured by the simulations [Figs. 5(c) and 5(d)] but in marked contrast to the measurements where $\omega_{0}$ remains small as $B \rightarrow 0$. This discrepancy points to a key difference between real ASIs, which are composed of mesoscopic superparamagnets that possess an intrinsic magnetic anisotropy barrier, and standard Monte Carlo models of binary spin systems, which typically do not include such intrinsic barriers. Kinetics in real ASIs are a nontrivial convolution of many-body timescales (which are simulated) and local timescales associated with magnetic anisotropy and dynamics within the islands themselves (which typically are not simulated). More realistic simulations that incorporate the details of magnetic anisotropy and magnetization reversal at the level of individual nanoislands is a topic of significant current interest [45], and we anticipate that future efforts along these lines will accurately capture the kinetics of ASIs that are now experimentally measurable using dynamic probes such as noise spectroscopy. Supplemental Material Fig. S6 shows that even a very simple incorporation of a superparamagnetic energy barrier into a standard Monte Carlo simulation of square ASI at zero field yields noise spectra with slower dynamics, qualitatively consistent with the noise data. 

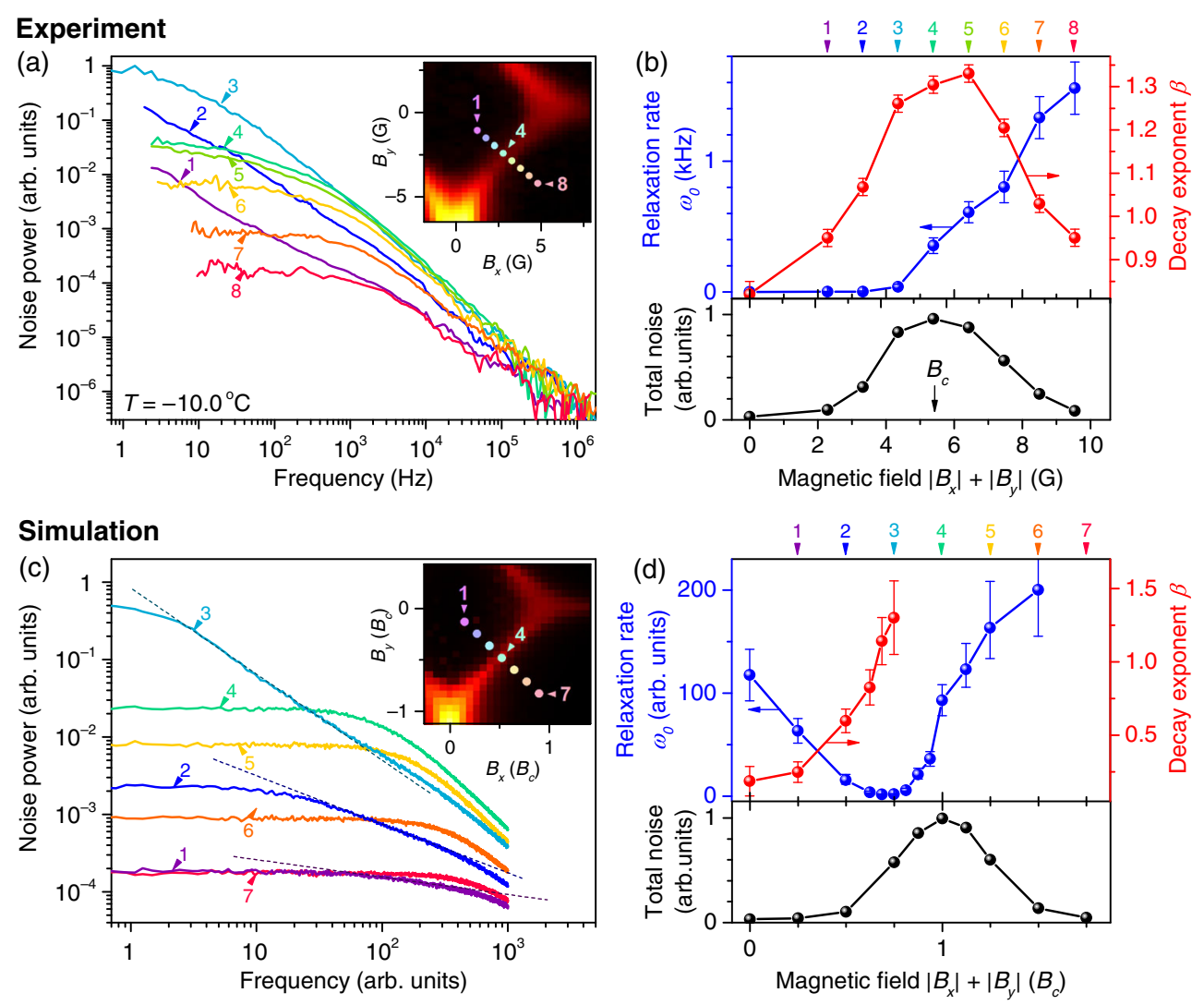

FIG. 5. Magnetization noise spectra through the monopole plasma regime. (a) Measured noise spectra along the indicated path (keeping $\left|B_{x}\right|=\left|B_{y}\right|$ ), which traverses between type-I and type-II order. Here, $T=-10.0^{\circ} \mathrm{C}$. (b) Extracted relaxation rates $\omega_{0}$ (blue points), decay exponents $\beta$ (red points), and total noise power (black points). Note that $\beta$ exhibits a maximum and is closest to a value of 2 when $B=B_{c}$, indicating that the monopoles exhibit their most-uncorrelated (most-diffusive) dynamics in the plasmalike regime where their density is largest. (c,d) Corresponding computed noise spectra from Monte Carlo simulations and extracted parameters $\omega_{0}$, $\beta$, and total noise. Dashed lines are guides to the eye, highlighting power-law decays. The total integrated noise is peaked when type-I and type-II vertex energies are degenerate, i.e., in the monopole plasma regime that occurs at the crossover field $B_{c}$. By contrast, simulations show that $\omega_{0}$ exhibits a minimum when the ASI orders antiferromagnetically (spontaneous type-I ordering), which occurs at an applied field that is slightly less than $B_{c}$ when $T>0$ (see also Supplemental Material Fig. S5 [26]).

The calculated minimum in $\omega_{0}$ highlights a further important distinction: The antiferromagnetic phase transition in square ASI [46] occurs at $B<B_{c}$ when $T>0$ (as mandated by the system's temperature-dependent free energy), whereas the monopole plasma always occurs at $B_{c}$-independent of temperature-because it is determined solely by the energy degeneracy of type-I and type-II vertices and the associated vanishing of the Dirac string tension. This distinction is apparent in the separation between $B_{c}$ and the minimum in $\omega_{0}$ in Fig. 5(d), and it is further elucidated at other temperatures by simulations of the specific heat and the antiferromagnetic order parameter shown in Supplemental Material Fig. S5 [26].

In summary, broadband noise spectroscopy introduces a new paradigm to ASI studies by providing a probe that is explicitly sensitive to dynamic timescales and correlations over many orders of magnitude in frequency. These results open the door to direct exploration of field or temperature phase diagrams and their intrinsic equilibrium dynamics, and the discovery of a field-tunable monopole plasma regime in archetypal square ASI demonstrates the power of such investigations. The ability to create monopole-rich regimes on demand - with tunable kinetic correlationssuggests the natural next steps of engineering monopole populations in finite-size arrays and in different lattice geometries. The additional availability of a widebandwidth dynamic probe opens the possibility of studying new regimes of magnetic charge dynamics in the more highly frustrated kagome systems, as well as the dynamics of the topological excitations recently demonstrated in vertex-frustrated ASIs [7]. As a long-term prospect, the ability to field-tune the presence or absence of magnetic charges suggests the possibility of transistorlike devices based on monopole flow, realizing new potential applications for these emergent effective charges.

We gratefully acknowledge support from the Los Alamos Laboratory Directed Research and Development 
(LDRD) program and discussions with Clare Yu, Herve Carruzzo, and Gia-Wei Chern. Work at the National High Magnetic Field Lab was supported by the National Science Foundation (NSF) Grant No. DMR-1644779, the State of Florida, and the U.S. Department of Energy. Work at Yale University was funded by the U.S. Department of Energy, Office of Basic Energy Sciences, Materials Sciences and Engineering Division under Grants No. DE-SC0010778 and No. DE-SC0020162. Work at the University of Minnesota was supported by the NSF through Grant No. DMR-1807124.

\section{APPENDIX: METHODS}

Sample fabrication.-Thermally active ASI lattices were fabricated by methods similar to those employed in prior work $[24,47]$. Briefly, electron beam lithography was used to pattern bilayer resist masks on $\mathrm{Si} / \mathrm{SiN}$ substrates for subsequent metal deposition and lift-off. Islands of lateral dimension $220 \mathrm{~nm} \times 80 \mathrm{~nm}$ were formed, with thicknesses of about $3.5 \mathrm{~nm}$. Ultrahigh vacuum (about $10^{-10}$ Torr base pressure and about $10^{-9}$ Torr deposition pressure) electron beam evaporation at $0.05 \mathrm{~nm} / \mathrm{s}$ was used for permalloy $\left(\mathrm{Ni}_{0.8} \mathrm{Fe}_{0.2}\right)$ deposition, in a molecular beam epitaxy system. The islands were then capped with two layers of thermally oxidized $\mathrm{Al}$ (total thickness of about $3 \mathrm{~nm}$ ) to minimize oxidation of the permalloy. Magneto-optical Kerr effect (MOKE) studies of noninteracting control samples confirmed that the islands were thermally active near room temperature, with typical fluctuation frequencies on the order of 1-10 kHz. Furthermore, MOKE studies of square ASI arrays confirmed that characteristic equilibration timescales (in response to, e.g., changing field or temperature) were fast, on the order of tens of milliseconds, ensuring that the ASI samples were in thermal equilibrium during the noise measurements.

Broadband magnetization noise spectroscopy.-The ASI samples were mounted face-up in the $x-y$ plane, on a positioning stage that could be temperature controlled from $-10^{\circ} \mathrm{C}$ to $+30^{\circ} \mathrm{C}$. The horizontal and vertical islands were oriented along $\hat{x}$ and $\hat{y}$, respectively. The magnetooptical noise spectrometer was adapted from an instrument previously developed to measure out-of-plane magnetization fluctuations [48]. A weak probe laser (less than $1 \mathrm{~mW}$ ), incident in the $x$ - $z$ plane, was linearly polarized and focused to a small (about $4 \mu \mathrm{m}$ diameter) spot on the ASI at $45^{\circ}$ incidence; therefore, about 300 islands were probed. Thermodynamic magnetization fluctuations along the $\hat{x}$ direction, $\delta m_{x}(t)$ (i.e., fluctuations of the horizontal islands only), imparted small Kerr rotation fluctuations $\delta \theta_{K}(t)$ on the polarization of the reflected laser, which were detected with balanced photodiodes. The magnetization noise was amplified and digitized, and its power spectrum was computed and signal-averaged in real time using fast Fourier transform (FFT) methods. Small coils were used to apply magnetic fields $B_{x}$ and $B_{y}$ in the sample plane.
The spectral density of the measured noise contained additional contributions from amplifier noise and photon shot noise, which were unrelated to, and uncorrelated with, the magnetization fluctuations from the ASI. We subtracted these constant contributions by also measuring the noise spectra in the presence of a large applied magnetic field $\left(B_{x}=B_{y} \simeq 20 \mathrm{G}\right)$, where all the islands were strongly polarized and magnetization noise from the ASI was entirely suppressed. Supplemental Fig. S2 shows raw time-domain noise data from square ASI at different magnetic fields.

To obtain the maps of the total (frequency-integrated) noise power vs applied magnetic field, for each value of magnetic field, the noise spectrum was acquired for several seconds, which allowed us to record good quality data in the frequency range from a few hundred $\mathrm{Hz}$ to a few hundred kHz. For more detailed studies of the noise spectra over the broadest possible frequency range (shown, for example, in Fig. 5), the measured noise was signalaveraged for a longer time duration (typically many tens of minutes), which increased the usable bandwidth from about $1 \mathrm{~Hz}$ to over $1 \mathrm{MHz}$.

Monte Carlo (MC) spin dynamics simulations.-We performed standard Glauber MC simulations of conventional square ASI lattices with periodic boundary conditions. We used single-spin updates (i.e., no cluster or loop moves), which should coarsely resemble the kinetics of the nanoislands in square ASI. Spins were chosen randomly, and the acceptance probability was $p=[1+\exp (\Delta / k T)]^{-1}$, where $\Delta$ is the usual energy difference associated with a spin flip and $k$ is the Boltzmann constant. Typical simulations utilized about $10^{6}$ annealing steps, and then the magnetization was recorded for up to several millions of MC time steps at fixed temperature $T$ and applied field $B$. Noise spectra were computed directly from the time series via fast Fourier transform.

The simulations employed a vertex model where the energetics were obtained from two energy scales: the nearest-neighbor coupling $J_{1}$ between perpendicular spins, and the weaker next-nearest-neighbor coupling $J_{2}$ between collinear spins. The simulations used $J_{1} / J_{2}=1.8$, in line with previous micromagnetic simulations of ASI systems, for which the ratio varies from 1.4 to 2.0 (depending on fabrication details). Within this model, the energies of the four different vertex topologies in zero applied magnetic field were $\epsilon_{\mathrm{I}}=-4 J_{1}+2 J_{2}, \epsilon_{\mathrm{II}}=-2 J_{2}$, and $\epsilon_{\mathrm{III}}=0$, $\epsilon_{\mathrm{IV}}=4 J_{1}+2 J_{2}$. The vertex model clearly defines the applied in-plane magnetic fields at which the monopole plasma regime is realized-namely, fields at which type-I and type-II vertices have equal energies: $\left|B_{x}\right|+\left|B_{y}\right| \propto$ $\epsilon_{\text {II }}-\epsilon_{\mathrm{I}}=4\left(J_{1}-J_{2}\right)$. Supplemental Material Fig. S1 [26] shows the origin of the diamond-shaped noise maps and a plot of all the different vertex energies as a function of applied magnetic field.

The simulation temperature $T$ was defined in units of $J_{2} / k$. Using $J_{1} / J_{2}=1.8$, the critical temperature $T_{c}$ below 
which square ASI spontaneously orders into its long-range type-I antiferromagnetic state was $T_{c} \simeq 2.4 J_{2} / k$ (at zero applied magnetic field). To most closely match experimental conditions, MC simulations were typically performed at lower temperatures, in the range of $T=1.4-1.8 J_{2} / k$. Supplemental Material Fig. S5 [26] shows additional simulations of the $T$ - and $B$-dependent antiferromagnetic order parameter and specific heat in thermal square ASI, in relation to the ( $T$-independent) monopole plasma regime. The applied fields $B_{x}$ and $B_{y}$ were defined in terms of the Zeeman energy on a single spin, and thus also in units of $J_{2}$. With these conventions, the monopole plasma was expected at $\left|B_{x}\right|+\left|B_{y}\right|=B_{c}=3.2 J_{2}$.

[1] S. H. Skjaervø, C. H. Marrows, R. L. Stamps, and L. J. Heyderman, Advances in Artificial Spin Ice, Nat. Rev. Phys. 2, 13 (2020).

[2] N. Rougemaille and B. Canals, Cooperative Magnetic Phenomena in Artificial Spin Systems: Spin Liquids, Coulomb Phase and Fragmentation of Magnetism, Eur. Phys. J. B 92, 62 (2019).

[3] I. Gilbert, C. Nisoli, and P. Schiffer, Frustration by Design, Phys. Today 69, No. 7, 54 (2016).

[4] R. F. Wang et al., Artificial "Spin Ice" in a Geometrically Frustrated Lattice of Nanoscale Ferromagnetic Islands, Nature (London) 439, 303 (2006).

[5] M. J. Harris, S. T. Bramwell, D. F. McMorrow, T. Zeiske, and K. W. Godfrey, Geometrical Frustration in the Ferromagnetic Pyrochlore $\mathrm{Ho}_{2} \mathrm{Ti}_{2} \mathrm{O}_{7}$, Phys. Rev. Lett. 79, 2554 (1997).

[6] S. T. Bramwell and M. J. Harris, The History of Spin Ice, J. Phys. Condens. Matter 32, 374010 (2020).

[7] C. Nisoli, V. Kapaklis, and P. Schiffer, Deliberate Exotic Magnetism via Frustration and Topology, Nat. Phys. 13, 200 (2017).

[8] A. Farhan et al., Nanoscale Control of Competing Interactions and Geometrical Frustration in a Dipolar Trident Lattice, Nat. Commun. 8, 995 (2017).

[9] J. Drisko, T. Marsh, and J. Cumings, Topological Frustration of Artificial Spin Ice, Nat. Commun. 8, 14009 (2017).

[10] Y.-L. Wang, Z.-L. Xiao, A. Snezhko, J. Xu, L. E. Ocola, R. Divan, J. E. Pearson, G. W. Crabtree, and W.-K. Kwok, Rewritable Artificial Magnetic Charge Ice, Science 352, 962 (2016).

[11] C. Castelnovo, R. Moessner, and S. L. Sondhi, Magnetic Monopoles in Spin Ice, Nature (London) 451, 42 (2008).

[12] S. T. Bramwell, S. R. Giblin, S. Calder, R. Aldus, D. Prabhakaran, and T. Fennell, Measurement of the Charge and Current of Magnetic Monopoles in Spin Ice, Nature (London) 461, 956 (2009).

[13] L. A. Mól, R. L. Silva, R. C. Silva, A. R. Pereira, W. A. Moura-Melo, and B. V. Costa, Magnetic Monopole and String Excitations in Two-Dimensional Spin Ice, J. Appl. Phys. 106, 063913 (2009).

[14] S. Ladak, D. E. Read, G. K Perkins, L. F. Cohen, and W. R. Branford, Direct Observation of Magnetic Monopole
Defects in an Artificial Spin-Ice System, Nat. Phys. 6, 359 (2010).

[15] E. Mengotti, L. J. Heyderman, A. F. Rodríguez, F. Nolting, R. V. Hügli, and H.-B. Braun, Real-Space Observation of Emergent Magnetic Monopoles and Associated Dirac Strings in Artificial Kagome Spin Ice, Nat. Phys. 7, 68 (2011).

[16] C. Phatak, A. K. Petford-Long, O. Heinonen, M. Tanase, and M. De Graef, Nanoscale Structure of the Magnetic Induction at Monopole Defects in Artificial Spin-Ice Lattices, Phys. Rev. B 83, 174431 (2011).

[17] A. Farhan, P. M. Derlet, A. Kleibert, A. Balan, R. V. Chopdekar, M. Wyss, J. Perron, A. Scholl, F. Nolting, and L. J. Heyderman, Direct Observation of Thermal Relaxation in Artificial Spin Ice, Phys. Rev. Lett. 111, 057204 (2013).

[18] X. M. Chen et al., Spontaneous Magnetic Superdomain Wall Fluctuations in an Artificial Antiferromagnet, Phys. Rev. Lett. 123, 197202 (2019).

[19] S. A. Morley et al., Thermally and Field-Driven Mobility of Emergent Magnetic Charges in Square Artificial Spin Ice, Sci. Rep. 9, 15989 (2019).

[20] J. P. Morgan, A. Stein, S. Langridge, and C. H. Marrows, Thermal Ground-State Ordering and Elementary Excitations in Artificial Magnetic Square Ice, Nat. Phys. 7, 75 (2011).

[21] Z. Budrikis, K. L. Livesey, J. P. Morgan, J. Akerman, A. Stein, S. Langridge, C. H. Marrows, and R. L. Stamps, Domain Dynamics and Fluctuations in Artificial Square Ice at Finite Temperatures, New J. Phys. 14, 035014 (2012).

[22] V. Kapaklis, U. B. Arnalds, A. Farhan, R. V. Chopdekar, A. Balan, A. Scholl, L. J. Heyderman, and B. Hjörvarsson, Thermal Fluctuations in Artificial Spin Ice, Nat. Nanotechnol. 9, 514 (2014).

[23] L. Anghinolfi, H. Luetkens, J. Perron, M. G. Flokstra, O. Sendetskyi, A. Suter, T. Prokscha, P. M. Derlet, S. L. Lee, and L. J. Heyderman, Thermodynamic Phase Transitions in a Frustrated Magnetic Metamaterial, Nat. Commun. 6, 8278 (2015).

[24] S. Zhang, I. Gilbert, C. Nisoli, G.-W. Chern, M. J. Erickson, L. O'Brien, C. Leighton, P. E. Lammert, V. H. Crespi, and P. Schiffer, Crystallites of Magnetic Charges in Artificial Spin Ice, Nature (London) 500, 553 (2013).

[25] E. Y. Vedmedenko, Dynamics of Bound Monopoles in Artificial Spin Ice: How to Store Energy in Dirac Strings, Phys. Rev. Lett. 116, 077202 (2016).

[26] See Supplemental Material at http://link.aps.org/ supplemental/10.1103/PhysRevX.11.011042 for Figs. S1S6.

[27] Y. Perrin, B. Canals, and N. Rougemaille, Extensive Degeneracy, Coulomb Phase and Magnetic Monopoles in Artificial Square Ice, Nature (London) 540, 410 (2016).

[28] A. Farhan et al., Emergent Magnetic Monopole Dynamics in Macroscopically Degenerate Artificial Spin Ice, Sci. Adv. 5, eaav6380 (2019).

[29] R. Dusad, F. K. K. Kirschner, J. C. Hoke, B. R. Roberts, A. Eyal, F. Flicker, G. M. Luke, S. J. Blundell, and J. C. Séamus Davis, Magnetic Monopole Noise, Nature (London) 571, 234 (2019). 
[30] I. A. Ryzhkin, Magnetic Relaxation in Rare-Earth Oxide Pyrochlores, J. Exp. Theor. Phys. 101, 481 (2005).

[31] A. V. Klyuev, M. I. Ryzhkin, A. V. Yakimov, and B. Spagnolo, Memory Effect and Generation-Recombination Noise of Magnetic Monopoles in Spin Ice, J. Stat. Mech. (2019) 094005.

[32] L. Bovo, J. A. Bloxsom, D. Prabhakaran, G. Aeppli, and S. T. Bramwell, Brownian Motion and Quantum Dynamics of Magnetic Monopoles in Spin Ice, Nat. Commun. 4, 1535 (2013).

[33] R. Kubo, Response, Relaxation and Fluctuation, in Transport Phenomena, Lecture Notes in Physics, edited by G. Kirczenow and J. Marro (Springer-Verlag, Berlin, 1974), Vol. 31, pp. 74-124.

[34] H. Mori, Transport, Collective Motion, and Brownian Motion, Prog. Theor. Phys. 33, 423 (1965).

[35] B. B. Mandelbrot and J. W. Van Ness, Fractional Brownian Motions, Fractional Noises and Applications, SIAM Rev. 10, 422 (1968).

[36] R. Saletti, A Comparison between Two Methods to Generate $1 / f^{\gamma}$ Noise, Proc. IEEE 74, 1595 (1986).

[37] B. Kaulakys, V. Gontis, and M. Alaburda, Point Process Model of $1 / f$ Noise vs a Sum of Lorentzians, Phys. Rev. E 71, 051105 (2005).

[38] J. Klafter, A. Blumen, and M. F. Shlesinger, Stochastic Pathway to Anomalous Diffusion, Phys. Rev. A 35, 3081 (1987).

[39] R. Metzler and J. Klafter, The Random Walks Guide to Anomalous Diffusion: A Fractional Dynamics Approach, Phys. Rep. 339, 1 (2000).
[40] J.-P. Bouchaud and A. Georges, Anomalous Diffusion in Disordered Media: Statistical Mechanisms, Models and Physical Applications, Phys. Rep. 195, 127 (1990).

[41] S. Havlin and D. Ben-Avraham, Diffusion in Disordered Media, Adv. Phys. 36, 695 (1987).

[42] S. C. Kou and X. S. Xie, Generalized Langevin Equation with Fractional Gaussian Noise: Subdiffusion within a Single Protein Molecule, Phys. Rev. Lett. 93, 180603 (2004).

[43] P. C. Hohenberg and B. I. Halperin, Theory of Dynamic Critical Phenomena, Rev. Mod. Phys. 49, 435 (1977).

[44] Z. Chen and C. C. Yu, Measurement-Noise Maximum as a Signature of a Phase Transition, Phys. Rev. Lett. 98, 057204 (2007).

[45] S. Koraltan et al., Dependence of Energy Barrier Reduction on Collective Excitations in Square Artificial Spin Ice: A Comprehensive Comparison of Simulation Techniques, Phys. Rev. B 102, 064410 (2020).

[46] O. Sendetskyi, V. Scagnoli, N. Leo, L. Anghinolfi, A. Alberca, J. Lüning, U. Staub, P. M. Derlet, and L. J. Heyderman, Continuous Magnetic Phase Transition in Artificial Square Ice, Phys. Rev. B 99, 214430 (2019).

[47] I. Gilbert, Y. Lao, I. Carrasquillo, L. O’Brien, J. D. Watts, M. Manno, C. Leighton, A. Scholl, C. Nisoli, and P. Schiffer, Emergent Reduced Dimensionality by Vertex Frustration in Artificial Spin Ice, Nat. Phys. 12, 162 (2016).

[48] A. L. Balk, F. Li, I. Gilbert, J. Unguris, N. A. Sinitsyn, and S. A. Crooker, Broadband Spectroscopy of Thermodynamic Magnetization Fluctuations through a Ferromagnetic SpinReorientation Transition, Phys. Rev. X 8, 031078 (2018). 